\title{
A!
}

This is an electronic reprint of the original article.

This reprint may differ from the original in pagination and typographic detail.

Davidovich, Michael V.; Nefedov, Igor S.

\section{Space-time dispersion and waveguide properties of 2D periodic metallic rod photonic crystals}

\section{Published in:}

Saratov Fall Meeting 2013: Optical Technologies in Biophysics and Medicine XV; and Laser Physics and Photonics XV

DOI:

$10.1117 / 12.2051860$

Published: 01/01/2014

Document Version

Publisher's PDF, also known as Version of record

Please cite the original version:

Davidovich, M. V., \& Nefedov, I. S. (2014). Space-time dispersion and waveguide properties of 2D periodic metallic rod photonic crystals. In E. A. Genina, V. L. Derbov, I. Meglinski, \& V. V. Tuchin (Eds.), Saratov Fall Meeting 2013: Optical Technologies in Biophysics and Medicine XV; and Laser Physics and Photonics XV [903115] (Proceedings of SPIE : the International Society for Optical Engineering; No. 9031). SPIE. https://doi.org/10.1117/12.2051860

This material is protected by copyright and other intellectual property rights, and duplication or sale of all or part of any of the repository collections is not permitted, except that material may be duplicated by you for your research use or educational purposes in electronic or print form. You must obtain permission for any other use. Electronic or print copies may not be offered, whether for sale or otherwise to anyone who is not an authorised user. 


\section{Space-time dispersion and waveguide properties of 2D periodic metallic rod photonic crystals}

Michael V. Davidovich, Igor S. Nefedov

Michael V. Davidovich, Igor S. Nefedov, "Space-time dispersion and waveguide properties of 2D periodic metallic rod photonic crystals," Proc. SPIE 9031, Saratov Fall Meeting 2013: Optical Technologies in Biophysics and Medicine XV; and Laser Physics and Photonics XV, 903115 (30 January 2014); doi: 10.1117/12.2051860

SPIE. Event: Saratov Fall Meeting 2013, 2013, Saratov, Russian Federation 


\title{
Space-time dispersion and waveguide properties of 2D-periodic metallic rod photonic crystals
}

\author{
Mikhael V. Davidovich ${ }^{\mathrm{a}}$, Igor S. Nefedov ${ }^{\mathrm{b}}$ \\ ${ }^{a}$ Department of Physics, Radio Engineering and Electromagnetics, Saratov State University, \\ Astrakhanskaya str., 83, Saratov, 410012, Russia \\ ${ }^{b}$ Department of Radio Science and Engineering, School of Electrical Engineering, Aalto University, \\ P.O. Box 13000, 00076 Aalto, Finland
}

\begin{abstract}
Metamaterials, made in form of periodically arranged metal and dielectric cylindrical inclusions, have been investigated on the basis of the integral equation method, based on periodic Green's function. Metal rods are described in terms of complex permittivity $\widetilde{\varepsilon}^{\prime}<0$. Along the rods terahertz and infrared waves propagate substantially with the speed of light $c$ and small losses weakly depending on the transverse wave number, whereas in the optical range, in particular in the shortwave part of a spectrum, they turn into the slow -waves of a dielectric waveguide.
\end{abstract}

Keywords: Photonic crystals, hyperbolic metamaterials, integral equations, spatial dispersion, wire media

\section{INTRODUCTION}

Waveguide properties and dispersion in a variety of perfectly conducting and impedance two-dimensional periodic pin (wire) structures (see Fig. 1) have recently been investigated [1-18]. Such structures are referred usually as metallic photonic crystals (PC) or wire media (WM). Were considered and studied conventional metal PC $[1-4,12,13,16]$, impedance structures [5, 14-15], periodic metamaterials based on carbon nanotubes and metallic nanowires [5-6] as well as based on ferromagnetic wire PC [10,11]. For these metamaterials different methods of homogenization were performed $[10,11,17-20]$. The greatest interest at the moment are the investigations of structures with dissipation, taking into account the real properties of metals in spectral ranges from the microwave to optical. Metal and metal-pin PC under certain conditions (at low frequencies and with low losses) exhibit properties of hyperbolic metamaterials (HM) [8, 21, 22]. These HM can be used in a number of applications: as artificial impedance and anisotropic surfaces [14, 21] (including supporting the propagation of Dyakonov's surface plasmons), for enhancement of radiative heat transfer [7,8], as good absorbers of radiation [20-23], for creation a lens imaging [24], and many other applications. Possible applications of HM are given in review [16].

Description of perfectly conducting or impedance pins (see Fig. 1) leads to a surface integral equation (IE), and in the case of thin pins to the linear IE $[12,13]$. 2D periodic structures arise on the assumption of a long wire length compared with other dimensions and a wavelength in vacuum. The most versatile and accurate method of describing these structures is the method of volume integral equations $[4,24,25]$ with the use of the periodic Green's functions (GF) [4-6, 14], which takes into account the internal field. This method has not been applied to analysis of HM.

Saratov Fall Meeting 2013: Optical Technologies in Biophysics and Medicine XV; and Laser Physics and Photonics XV, edited by Elina A. Genina, Vladimir L. Derbov, Igor Meglinski, Valery V. Tuchin, Proc. of SPIE Vol. 9031, 903115

(C) 2014 SPIE · CCC code: 1605-7422/14/\$18 - doi: 10.1117/12.2051860 


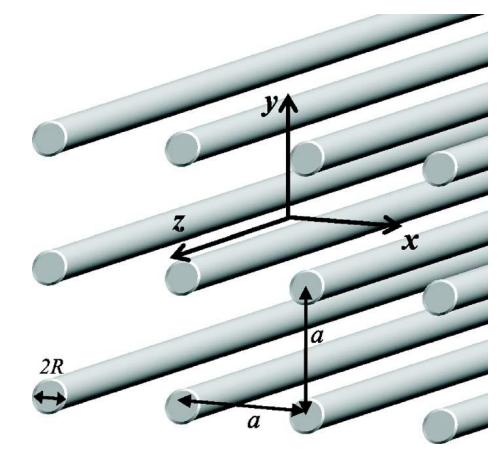

Figure 1. Schematic view of two-dimensional periodic wire medium with the square lattice.

First guiding properties of a metal wire were explored the Sommerfeld in $1899[26,27]$ in connection with the diffraction of a plane wave on the wire. Previously, he has assumed the presence of surface waves above the conductive structures [28]. The corresponding Sommerfeld-Zenneck wave is a slow, flowing into the wire [27] wave if the radius of wire is small in the range from radiofrequencies to microwave. The surface Zenneck wave (SZW) over the dissipative (conductive) plane can be both fast or slow [27]. The transition from the slow to fast SZW in the metal cylinder can occur at some ratio between the radius and the wavelength in the presence of significant losses. In the area of plasma frequency the SZW corresponds to the surface plasmon. Recently published an erroneous work [29] disproves the existence of the fast SZW that is a mistake because the wave flowing nature is not taken into account. In [30] we have proved the fallacy of conclusions [29] and have shown its flowing as in [27]. The term "flowing wave" along with all the "leaking waves" for classification of improper physical modes was used, for example, in [31] (see also [27]). Multi-wire structures canalize energy better than a single wire. Considered periodic HM, shown in Fig. 1, channel the energy along nanowires without cut-off for any transverse components of the wave vector. In the case of perfect conductivity the TEM-wave propagates along wires with the speed of light and also high-order fast waves. In the papers [6-9, 15] properties of metamaterials with different impedances rods have been studied. However, if do not take into account the internal field, the method of impedance IE, can give significant errors (especially for thin witres), as will be shown below.

It is interesting a rigorous investigation of waves and their homogenization (obtaining effective electrophysical parameters) in the frequency range from microwave to optical, as well as an establishment of applicability limits of previously obtained results. That is the objective of our work. To do this, we take into account the field inside the metal cylinder using a universal method of volume integral equations. Here this method is applied to 2D periodic PC formed of infinitely long metal nanorods with a square lattice in the transverse plane. Metal properties are described in terms of the complex permittivity [32].

\section{STATEMENT OF THE PROBLEM}

Time-space dependence of fields is taken to be $\exp (j \omega t-j \mathbf{k r})$ in 2D metal PC, embedded into a host matrix with the permittivity $\varepsilon$ and metal rods are characterized by the complex permittivity $\widetilde{\varepsilon}$. In terms of the effective medium theory the structure is described by a diagonal permittivity tensor $\hat{\varepsilon}$, where $\varepsilon_{x x}=\varepsilon_{y y}=\varepsilon$. In a lossless case fixing $k_{z}$ we can consider the transverse band structure in the $\left(k_{x} k_{y}\right)$ plane. The real part of $\varepsilon_{z z}$ may be negative, in this case wire media exhibit properties of HM $[8,21]$. We are interested in waves propagating along the rods as $\exp \left(j \omega t-j k_{z} z\right)$ at arbitrary $k_{x}$ and $k_{y}$. It makes our problem a two-dimensional in a WM cross-section and determines the spatial dispersion $k_{z}=\gamma\left(k_{x}, k_{y}\right)$. Let us assume the frequency to be fixed and omit the corresponding exponential factor. At low frequencies and in a lossless case, the wave is supported by surface currents, which can be replaced by linear ones along the wires, and dispersion equation is obtained using surface or linear IEs [4]. A more rigorous approach is based on the impedance IE [6] . Our full-wave analysis is based on the polarization current density 


$$
\mathbf{J}_{p}(\mathbf{r}, \omega)=j \omega \varepsilon_{0}(\widetilde{\varepsilon}(\omega)-\varepsilon) \mathbf{E}(\mathbf{r}, \omega) .
$$

It corresponds to a volume IE, which for a $z$-uniform waveguide structure is reduced to a two-dimensional crosssectional IE with respect to $\mathbf{E}\left(\mathbf{r}_{\perp}\right)$. The problem is solved in the approximation of thin wires $(r<<\lambda)$ and diluted PC $(r<<a)$ using periodic, i.e. Green's function of periodically spaced and phased sources, which allows us to consider only one component $J_{p z}$ which is nonzero only in that section, according to (1). Here $r$ is the radius of wires, $a$ - period of PC lattice, $\lambda$ is the wavelength. In accordance with (1), the volume IE for the electrical field is reduced to 2D IE with respect to the electric field component $E_{z}$ in the cross-section. Neglecting small transverse components of the electric field, the vector potential has a longitudinal component:

$$
A_{z}(x, y)=j \omega \varepsilon_{0}(\widetilde{\varepsilon}-\varepsilon) \int_{S} \widetilde{G}\left(\mathbf{r}_{\perp}-\mathbf{r}_{\perp}^{\prime}\right) E_{z}\left(\mathbf{r}_{\perp}^{\prime}\right) d^{2} r^{\prime}
$$

and determines $E_{z}$ as

$$
E_{z}(x, y)=\frac{\left(k^{2}-\gamma^{2}\right) A_{z}(x, y)}{j \omega \varepsilon_{0} \varepsilon}=\left(k^{2}-\gamma^{2}\right)(\widetilde{\varepsilon} / \varepsilon-1) \int_{S} \widetilde{G}\left(\mathbf{r}_{\perp}-\mathbf{r}_{\perp}^{\prime}\right) E_{z}\left(\mathbf{r}_{\perp}^{\prime}\right) d^{2} r^{\prime}
$$

where $S$ is the cross-section area, $k=k_{0} \sqrt{\varepsilon}=2 \pi \sqrt{\varepsilon} / \lambda$, and 2D Green's function reads as [3, 4]

$$
\widetilde{G}\left(\mathbf{r}_{\perp}-\mathbf{r}_{\perp}^{\prime}\right)=\frac{1}{a_{x} a_{y}} \sum_{m=-\infty}^{\infty} \sum_{n=-\infty}^{\infty} \frac{\exp \left(-j \tilde{k}_{x m}\left(x-x^{\prime}\right)-j \tilde{k}_{y n}\left(y-y^{\prime}\right)\right)}{\widetilde{k}_{x m}^{2}+\widetilde{k}_{y n}^{2}+\gamma^{2}-k^{2}}
$$

Here $\tilde{k}_{x m}=k_{x}+2 m \pi / a_{x}, \tilde{k}_{y m}=k_{y}+2 n \pi / a_{y}$ and we take $a_{x}=a_{x}=a$ in our numerical solution. Reduction of 3 D IEs to 2 D IEs is described in $[3,4,33]$. Below IE (3) will be refined taking into account the radial component $E_{\rho}$.

We solve the integral equation (3) choosing a coordinate system in null zone of PC in the center of the metal rod and going to cylindrical coordinates. Approximate IE (3) is the more precise the less $r / c$ since the lower transverse components $E_{\rho}$ and $E_{\varphi}$ the electric field in comparison with the longitudinal one. In the following calculations we take $\varepsilon=1$, i.e. $k=k_{0}$. Then we assume that $E_{z}(\rho, \varphi)$ does not depend on the angle $\varphi$. This is a very strong assumption. It means that the field on the pin surface is constant and varies only along the radius. Since the propagating wave attenuates, this assumption is possible with a small damping in the transverse direction at distances of the order $r$ and only if $\lambda>a$ that is possible with the radius of tens of nanometers and for sparse PC. Namely, the sparseness leads to relatively small losses. Dispersion equation is obtained multiplying (3) by $E_{z}(x, y)$ and integrating over the cross-section. Using the following relation in polar coordinates (see [3, 4])

$$
\frac{1}{2 \pi} \int_{0}^{2 \pi} \exp ( \pm j r(\alpha \cos (\varphi)+\beta \sin (\varphi))) d \varphi=J_{0}\left(r \sqrt{\alpha^{2}+\beta^{2}}\right)
$$

we obtain the functional

$$
\Phi\left(k_{0}, \mathbf{k}_{\perp}, \gamma, E_{z}\right)=\int_{0}^{r} E_{z}^{2}(\rho) d \rho-\left(k_{0}^{2}-\gamma^{2}\right)(\widetilde{\varepsilon}-1) \int_{0}^{r} \int_{0}^{r} E_{z}(\rho) K\left(\rho, \rho^{\prime}\right) E_{z}\left(\rho^{\prime}\right) \rho^{\prime} d \rho^{\prime} d \rho,
$$


with the kernel:

$$
K\left(\rho, \rho^{\prime}\right)=\frac{2 \pi}{a^{2}} \sum_{m=-\infty}^{\infty} \sum_{n=-\infty}^{\infty} \frac{J_{0}\left(\rho \tilde{\kappa}_{m n}\right) J_{0}\left(\rho^{\prime} \tilde{\kappa}_{m n}\right)}{\tilde{\kappa}_{m n}^{2}+\gamma^{2}-k_{0}^{2}}
$$

where $\widetilde{\kappa}_{m n}=\sqrt{\widetilde{k}_{x m}^{2}+\widetilde{k}_{y n}^{2}}$ and the dispersion equation reads as

$$
\Phi\left(k_{0}, \mathbf{k}_{\perp}, \gamma, \bar{E}_{z}\right)=0
$$

Here $\bar{E}_{z}$ is an unknown $z$-component of the electric field which is found using the method of moments expanding $E_{z}(\rho)$ of the basic functions. Then the following approximation is used. We assume that $E_{z}$ is azimuthally symmetric inside the rod, i.e. $E_{z}(\rho)=C_{1} J_{0}(\rho \kappa)$ where $C_{1}$ is unknown coefficient and $\kappa=\sqrt{k_{0}^{2} \widetilde{\varepsilon}-\gamma^{2}}$ In this approximation dispersion equation reads as

$$
\widetilde{\Phi}\left(k_{0}, \mathbf{k}_{\perp}, \gamma, E_{z}\right)=\int_{0}^{r} J_{0}^{2}(\rho \kappa) \rho d \rho-\left(k_{0}^{2}-\gamma^{2}\right)(\widetilde{\varepsilon}-1) \int_{0}^{r} \int_{0}^{r} \rho J_{0}(\rho \kappa) K\left(\rho, \rho^{\prime}\right) J_{0}\left(\rho^{\prime} \kappa\right) \rho^{\prime} d \rho^{\prime} d \rho=0 .
$$

Solving (9) numerically one can find unknown $\gamma$ as the function of transverse components of the wave vector in WM.

\section{NUMERICAL RESULTS}

The results presented below were obtained from the formula (9) using the iteration method. Figures 2 and 3 show the real and imaginary parts of the normalized propagation constant $\gamma$ versus the transverse components of the wave vector where the real and imaginary parts of the permittivity vary in a very wide range. These values correspond to different metals, semi-metals and semiconductors. In this case we took $k_{0} a=1$.
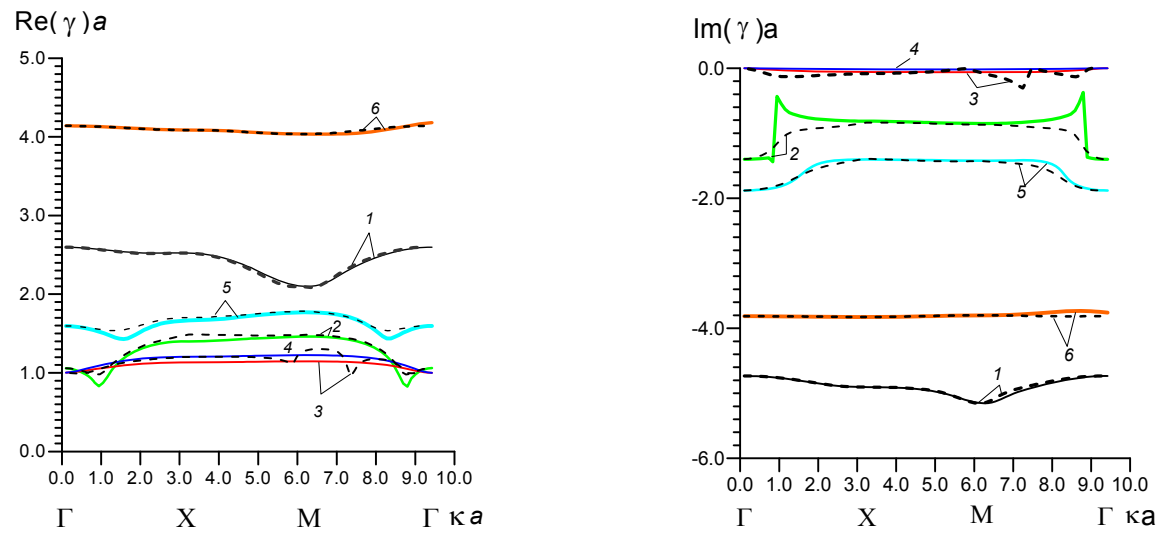

Figure 2. Normalized real (left) and imaginary (right) parts of the propagation constant, calculated for rods, characterized with different complex $\widetilde{\varepsilon}$ : $\widetilde{\varepsilon}=3-\mathrm{j} 10$ (curves 1); $\widetilde{\varepsilon}=-10-\mathrm{j} 100$ (curves 2); $\widetilde{\varepsilon}=-100-\mathrm{j} 1000$ (curves 3); $\widetilde{\varepsilon}=-900-\mathrm{j} 1000$ (curves 4); $\widetilde{\varepsilon}=-3-\mathrm{j} 50$ (curves 5); $\widetilde{\varepsilon}=-3-\mathrm{j} 10$ (curves 6). Solid and dashed curves correspond to real and complex $k_{x}, k_{y}$, respectively. 
Figures 3 show that gold wire media can transfer electromagnetic energy with small losses in the near-infrared and optical range and exhibit hyperbolic dispersion in these ranges.

\section{CONCLUSIONS}

Using the method of volumetric integral equations, formulated on the basis of periodic Green's function, we calculated electrophysical parameters of the simplest metal-dielectric PC with inclusions in the form of thin circular cylindrical rods, forming the two-dimensional periodic lattice. Rods are described by a complex macroscopic permittivity. The method can be easily generalized for rods, inhomogeneous along the radius, as well as for thin rods of finite length in three-dimensional periodic lattice. It was shown that wire media are able to effectively and practically without losses to transfer electromagnetic energy from the microwave until the infrared range with almost the speed of light. Noticeable losses and slowing occur only in the optical range.
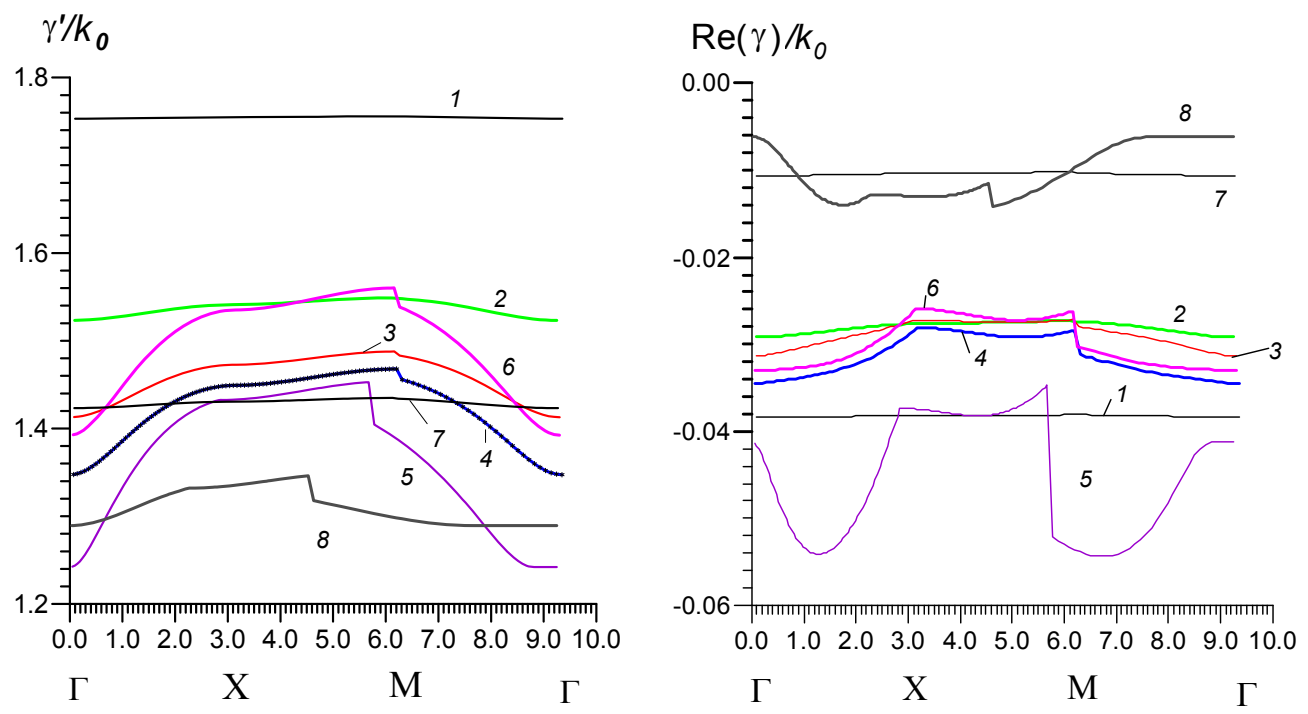

Figure 3. Normalized real (left) and imaginary (right) parts of the propagation constant for the quasi-TEM wave, propagating along gold wires. Curves $1-5$ correspond to the following sizes: $r=20 \mathrm{~nm}, a=500 \mathrm{~nm}$; curves 7,8 $r=40 \mathrm{~nm}, a=300 \mathrm{~nm} ; 6-r=20$ $\mathrm{nm}, a=300 \mathrm{~nm}$. Calculations were implemented at the following wavelengths: $\lambda=0.7 \mu \mathrm{m}$ (curves 1,7$) ; \lambda=1.0 \mu \mathrm{m}$ (curves $2,6,8)$; $\lambda=1.3 \mu \mathrm{m}$ (curve 3 ); $\lambda=1.5 \mu \mathrm{m}$ (curve 4 ); $\lambda=1.8 \mu \mathrm{m}$ (curve 5).

\section{REFERENCES}

[1] Belov, P. A., Marques, R., Maslovski, S. I., Nefedov, I. S., Silveirinha, M., Simovski, C. R. and Tretyakov, S. A., "Strong spatial dispersion in wire media in the very large wavelength limit," Phys. Rev. B 63, 113103 (2003).

[2] Nefedov, I. S., Viitanen, A. J. and Tretyakov, S. A., "Propagating and evanescent waves in two-dimensional wire media," Phys. Rev. E 71, 046612 (2005).

[3] Davidovich, M.V., "Photonic crystals: Green's functions, integro-differential equations, and simulation results," Radiophysics and Quantum Electronics 49, 134-145 (2005).

[4] Nefedov, I.S. and Viitanen, A. J. "Wire Media," in Metamaterials Handbook: Theory and Phenomena of Metamaterials, Ed. by F. Gapolino, Taylor \& Francis group, Boca Raton, London, New York, 15 (2009).

[5] Nefedov, I. S., "Electromagnetic waves propagating in a periodic array of parallel metallic carbon nanotubes," Phys. Rev. B 82, 155423 (2010).

[6] Nefedov, I. and Tretyakov, S., "Ultabroadband electromagnetically indefinite medium formed by aligned carbon nanotubes," Phys. Rev. B 84, 113410 (2011). 
[7] Nefedov, I. S. and Simovski, C. R., "Giant radiation heat transfer through micron gaps," Phys. Rev. B 84, 195459 (2011).

[8] Simovski, C., Maslovski, S., Nefedov, I. and Tretyakov, S., "Optimization of radiative heat transfer in hyperbolic metamaterials for thermophotovoltaic applications," Optics Express 21, 14988-15013 (2013).

[9] Nefedov, I. S. and Tretyakov, S. A., "Effective medium model for two-dimensional periodic arrays of carbon nanotubes," Photonics and Nanostructures - Fundamentals and Applications 9, 374-380 (2011).

[10] Liberal, I., Nefedov, I. S., Ederra, I., Gonzalo, R. and Tretyakov, S. A., "On the effective permittivity of arrays of ferromagnetic wires," J. Appl. Phys. 110, 104902 (2011).

[11]Liberal, I., Nefedov, I. S., Ederra, I., Gonzalo, R. and Tretyakov, S. A., "Electromagnetic response and homogenization of grids of ferromagnetic microwires," J. Appl. Phys. 110, 064909 (2011).

[12] Davidovich, M.V., Stephuk, J. V. and Shilovskii, P.A., "Electrophysical properties of metallic wire photonic crystals," Technical Physics 57, 320-327 (2012).

[13] Davidovich, M. V. and Shilovskii, P. A., "Calculation of the band diagrams of metallic wire photonic crystals," Technical Physics 57, 1687-1691 (2012).

[14] Davidovich, M. V. and Shilovskii, P. A., "Metamaterials with Dielectric and Metallic Inclusions in the Cubic Lattice," Technical Physics 58, 1173-1181 (2013).

[15] Liberal, I., Nefedov, I. S., Ederra, I., Gonzalo, R. and Tretyakov, S. A., "Reconfigurable Artificial Surfaces Based on Impedance Loaded Wires Close to a Ground Plane," IEEE Trans. on Antennas and Propag. 60, 19211930 (2012).

[16] Simovski, C.R., Belov, P.A., Atrashchenko, A.V. and Kivshar, Yu. S., "Wire metamaterials: Physics and Applications," Advanced Materials 23, 4229-4248 (2012).

[17] Davidovich, M. V. and Stephuk, J. V.. "Homogenization of periodic artificial media // Modeling in Applied Electromagnetics and Electronics," Saratov University Press, 67-75 (2007).

[18] Davidovich, M. V. and Stephuk, J. V., "Homogenization of periodic metamaterials // Mathematical Methods in Electromagnetic Theory," Proceedings of 12-th International Conference (MMET'2008). Odessa, Ukraine, 527-529 (2008).

[19] Guo, Y., Newman, W., Cortes, C. L. and Jacob, Z., "Applications of hyperbolic metamaterial substrates," Adv. Optoelectron., 1-9 (2012).

[20] Narimanov, E. E., Li, H., Barnakov, Yu. A., Tumkur, T. U. and Noginov, M. A., "Darker than black: radiationabsorbing metamaterial," arXiv:1109.5469v1 (2011).

[21] Hashemi, S. and Nefedov, I., "Wideband perfect absorption in arrays of tilted carbon nanotubes," Phys. Rev. B 86,195411 (2012).

[22] Nefedov, I. S., Valagiannopoulos, C. A., Hashemi, S. M. and Nefedov, E. I. "Total absorption in asymmetric hyperbolic media," Sc. Rep. 3, 2662 (2013).

[23] Nefedov, I. S., Valagiannopoulos, C. A. and Melnikov, L.A., "Perfect absorption in graphene multilayers," J. Opt. 15, 114003 (2013).

[24]Rahman, A., Kosulnikov, S.Yu., Hao, Y., Parini, C. and Belov, P.A., "Subwavelength optical imaging with array of silver nanoroads," J. Nanophoton. 5(1), 051601 (2011).

[25] Davidovich, M. and Stephuk, Yu., "Iteration methods and algorithms for dielectric-resonator integral equations of the field," Radiophysics and Quantum Electronics 53, 268-280 (2010).

[26] Sommerfeld, A., "Über die Fortpflanzung elektrodynamischer Wellen an längs eines Drahtes", Ann. der Physik und Chemie," 67, 233-290, (1899). Tr.: "Propagation of electro-dynamic waves along a cylindric conductor".

[27] Vainshtein, L. A., "Electromagnetic Waves," 2nd ed., Moscow: Radio i Svyaz' (1988).

[28] Sommerfeld, A., "Mathematische Theorie der Diffraction," Math. Ann. 47 317-374. (1896).

[29] Kukushkin, A. V., Rukhadze, A. A. and Rukhadze, K. Z., "On the existence conditions for a fast surface wave," Phys. Usp. 55, 1124-1133 (2012). 